\title{
Future Liver remnant: A Challenge In Liver Resection Surgery
}

Regmee S, Tamang TY, Maharjan DK, Shrestha SK, Thapa PB.

Department of Surgery, Kathmandu Medical College Teaching Hospital

Sinamangal, Kathmandu

Correspondence : Dr. Sujan Regmee, Resident, Department of Surgery, Kathmandu Medical College Teaching Hospital

Email sujanregmee@hotmail.com

\begin{abstract}
Introduction and Objective: The frontiers of liver resection are expanding. Future Liver Remnant; its volume, function and complication have a predictive value in post resection outcome including post resection liver failure. This study is directed to discuss the issues related to future liver remnant.

Objective: To discuss issues related to Future Liver Remnant in patients planned for major liver resection

Materials and Methods: Data of patients admitted for liver resection at Kathmandu Medical College Teaching Hospital, Surgical Unit III with varied diagnosis, were collected prospectively over a period of 1 year. In our non transplant center, Future Liver Remnant was managed with varying methods. Of the 12 liver resections, 3 prototype cases with different FLR issues are discussed in this study.

Results: The first case had issue related to the volume of the liver remnant for which she underwent a portal vein ligation followed by resection (after adequate volume increase) in the second surgery. The second case was a Hepatocellular Carcinoma with CTP 8 cirrhosis. Despite adequate liver volume, decision for liver resection was delayed due to possible postoperative decompensation. The third patient was a Hilar cholangiocarcinoma with obstructive jaundice. Biliary decompression (PTBD) was performed. However, inadequate fall in bilirubin caused dilemma for liver resection. Despite the dilemma, all 3 cases underwent liver resection with no postoperative liver failure.
\end{abstract}

Conclusion: Issues related to the volume and function of future liver remnant in patient undergoing liver resection should be adequately addressed by various pre operative, intra operative, and post operative measures.

Key words: Future liver remnant, volumetry, liver resection. 